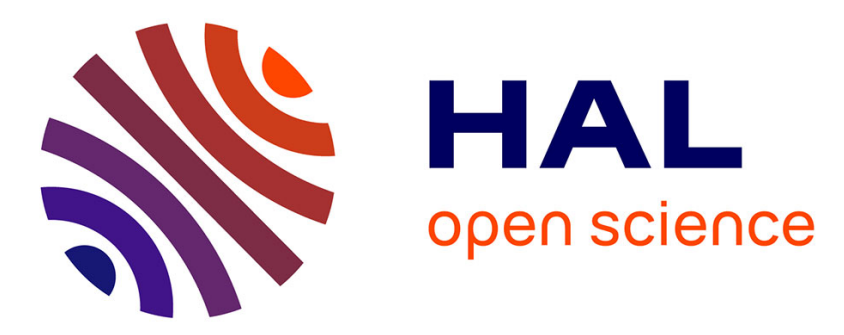

\title{
Frequency allocation problem in a SDMA satellite communication system
}

\author{
Laurent Houssin, Christian Artigues, Erwan Corbel
}

\section{To cite this version:}

Laurent Houssin, Christian Artigues, Erwan Corbel. Frequency allocation problem in a SDMA satellite communication system. Computers \& Industrial Engineering, 2011, 61 (2), pp.346-351. hal-00667946

\section{HAL Id: hal-00667946 \\ https://hal.science/hal-00667946}

Submitted on 8 Feb 2012

HAL is a multi-disciplinary open access archive for the deposit and dissemination of scientific research documents, whether they are published or not. The documents may come from teaching and research institutions in France or abroad, or from public or private research centers.
L'archive ouverte pluridisciplinaire HAL, est destinée au dépôt et à la diffusion de documents scientifiques de niveau recherche, publiés ou non, émanant des établissements d'enseignement et de recherche français ou étrangers, des laboratoires publics ou privés. 


\title{
Frequency allocation problem in a SDMA satellite communication system
}

\author{
Laurent Houssin $^{\mathrm{a}, \mathrm{b}, *}$, Christian Artigues ${ }^{\mathrm{a}, \mathrm{b}}$, Erwan Corbel $^{\mathrm{c}}$ \\ ${ }^{a}$ CNRS ; LAAS ; 7 avenue du colonel Roche, F-31077 Toulouse, France \\ ${ }^{b}$ Université de Toulouse ; UPS, INSA, INP, ISAE ; LAAS ; F-3107r7 Toulouse, France \\ ${ }^{c}$ Thales Alenia Space, Advanced Telecom 65 Navigation Systems Research, 26 av. J.F. \\ Champollion, 31037 Toulouse, France
}

\begin{abstract}
SDMA (Spatial Division Multiple Access) is a principle of radio resource sharing that relies on the division of the space dimension into separated communication channels. SDMA basically relies on adaptive and dynamic beam-forming associated to a clever algorithm in charge of resource allocation. As satellite communication systems move towards an increasing number of users and a larger throughput for each of them, SDMA is one of the most promising techniques that can reach these two goals. This paper studies static Frequency Assignment Problems (FAP) in a satellite communication system involving a gateway connected to a terrestrial network and some user terminals located in a service area. Two scenarios are considered: one based on SDMA and the other based on usual spot coverage. We propose original integer linear programming formulations and greedy allocation algorithms for the FAP which involves unusual cumulative interference constraints. By considering the link budget of each user, the objective is to maximize the number of users that the system can serve. We show through computational experiments on realistic data that the FAP associated with the SDMA system can be solved efficiently, yielding substantial improvement compared to the traditional system.
\end{abstract}

Keywords: SDMA system, radio resource management, frequency assignment problem, integer programming, greedy algorithms

\footnotetext{
* Corresponding author

Email addresses: houssin@laas.fr (Laurent Houssin), artigues@laas.fr (Christian Artigues), erwan.corbel@thalesaleniaspace.com (Erwan Corbel)
} 


\section{Introduction}

Satellite communication systems move towards greater capacity, higher flexibility (with respect to the position of the users) and better service to the end-user. SDMA (Spatial Division Multiple Access) appears to be one way to achieve these requirements at the same time Liberti and Rappaport (1999). SDMA is a principle of radio resource sharing that relies on the division of the space dimension into separated communication channels. It can be used with common Frequency Division Multiple Access (FDMA), Time Division Multiple Access (TDMA) or Code Division Multiple Access (CDMA) techniques. Main future terrestrial communication standards (such that WIMAX, 3GPP, LTE) implement SDMA.

Today SDMA is currently used by IRIDIUM system in L-band, a constellation of 66 Low-Earth Orbit satellites, thanks to time beam-switching. SDMA is also foreseen as a key enabling technique to increase the capacity of future two-way satellite communications systems in low-frequency bands (typically lower than 5-6 GHz) through the interference mitigation and high frequency reuse Corbel et al. (2008). It is also expected to play an important role in future systems devoted to Public Protection and Disaster Relief (PPDR) and Global Monitoring for Environment and Security (GMES) missions which require on-demand beam-forming Gayrard et al. (2007).

SDMA basically relies on adaptive and dynamic beam-forming associated to a clever algorithm in charge of resource allocation. The satellite beamformer optimizes the antenna diagram with respect to the positions of the users in order to maximize the gain while mitigating interferences. The resource allocation algorithm carefully designs a frequency plan that

- prevents or limits interferences between users,

- tailors the allocated bandwidth to the user need in order to save the spectrum.

The spatial filtering capability of adaptive antenna can be used to implement SDMA in communication systems where users share the same channels. Moreover, the resource management by spatial filtering of users give the opportunity to massively reuse radio channels. It makes the channel allocation 
strategy play an important role in the system performance. This class of problem is well known as Frequency Assignment Problem (FAP).

The satellite telecommunication system that we study in this paper aims at establishing bi-directional communications involving a gateway connected to a terrestrial network and some user terminals located in a service area. This paper studies static frequency allocation problems in this system and two scenarios are considered: one based on SDMA and the other based on "traditional" spot coverage. We propose original Integer Linear Programming (ILP) formulations and greedy allocation algorithms for these problems. The difficulty for solving the FAP is increased by considering cumulative interference constraints. We then compare the performance of the two scenarios.

The remainder of the paper is organized as follow: Section 2 is dedicated to the telecommunication system and the description of the scenarios. In Section 3, we present a cumulative formulation of the FAP interference constraints. In Section 4, ILP formulations and greedy algorithms proposed for both scenarios are described. Section 5 presents the results obtained by the different algorithms on the scenarios. Concluding remarks are drawn in Section 6.

\section{Telecommunication system and scenarios for frequency alloca- tion}

\subsection{System description}

The service area is a rectangular grid where users position is uniformly distributed and where beams are directed. The grid size is $u=[-0.043980,0.048870]$ and $v=[-0.021152,0.012702]$ (Cartesian coordinates are considered although the same study can be done with spherical coordinates) with a step of $5 \times 10^{-4}$. The satellite orthogonal projection onto the service area defines point $(0,0)$ of the grid.

Beams have two particular characteristics which are the direction that we consider through the position of the beam center in the service area, and the radiation pattern. An analytic representation of the radiation pattern enables to compute the directive gain of the antennas for a considered direction. This description is such that

$$
G_{\text {Sat }}\left(u, v, u_{0}, v_{0}\right)=G_{1} \times G_{2}\left(u, v, u_{0}, v_{0}\right) \times G_{3}\left(u, v, u_{0}, v_{0}\right)
$$


with

$$
\begin{gathered}
G_{1}=\eta\left(\frac{\pi D}{\lambda}\right)^{2}, \\
G_{2}\left(u, v, u_{0}, v_{0}\right)=\left(\frac{2 J_{1}\left(\frac{\pi D}{\lambda} \sqrt{\left(u-u_{0}\right)^{2}+\left(v-v_{0}\right)^{2}}\right)}{\frac{\pi D}{\lambda} \sqrt{\left(u-u_{0}\right)^{2}+\left(v-v_{0}\right)^{2}}}\right)^{2}
\end{gathered}
$$

and

$$
G_{3}(u, v)=\left(\frac{2 J_{1}\left(\frac{\pi d}{\lambda} \sqrt{u^{2}+v^{2}}\right)}{\frac{\pi d}{\lambda} \sqrt{u^{2}+v^{2}}}\right)^{2},
$$

where $J_{1}(x)$ is the Bessel functions of the first kind. A singular property of Bessel functions of the first kind is that $\frac{J_{1}(x)}{x}=1 / 2$ if $x=0$. It leads to $G_{2}\left(u, v, u_{0}, v_{0}\right)=1$ if $(u, v)=(u 0, v 0)\left(G_{2}\left(u, v, u_{0}, v_{0}\right)<1\right.$ otherwise $)$ and $G_{3}(u, v)=1$ if $(u, v)=(0,0)\left(G_{3}(u, v)<1\right.$ otherwise $)$. We use the following notations:

$u, v$ Cartesian coordinates of the user terminal;

$u_{0}, v_{0}$ Cartesian coordinates of the beam center;

$\eta$ antenna gain;

$D$ antenna diameter;

$d$ primary source diameter;

$\lambda$ wavelength.

The equation (1) was provided by the satellite company Thales Alenia Space. To the best of our knowledge, this equation has a particular shape and is not known in the literature. The term $G_{1}$ corresponds to the maximum gain antenna whereas $G_{2}$ depends on the distance between the user and the beam center, i.e.

$\sqrt{\left(u-u_{0}\right)^{2}+\left(v-v_{0}\right)^{2}}$ and $G_{3}$ depends on user position related to the satellite, i.e. $\sqrt{u^{2}+v^{2}}$. The product $G_{2}\left(u, v, u_{0}, v_{0}\right) \times G_{3}\left(u, v, u_{0}, v_{0}\right)$ is illustrated by a $3 \mathrm{D}$ representation in fig. 1 and by a $2 \mathrm{D}$ representation in fig. 2 where the left pattern is the pattern of beam centered in $(0.03,-0.03)$ and the right one is centered in $(0,0)$, i.e directly under the satellite. It emphasizes that the more the beam center is closed to the satellite, the more the gain is high. We 
can also remark in fig.2 that closed to the sharp summit, small waves exist and will disturb users that share the same frequency.

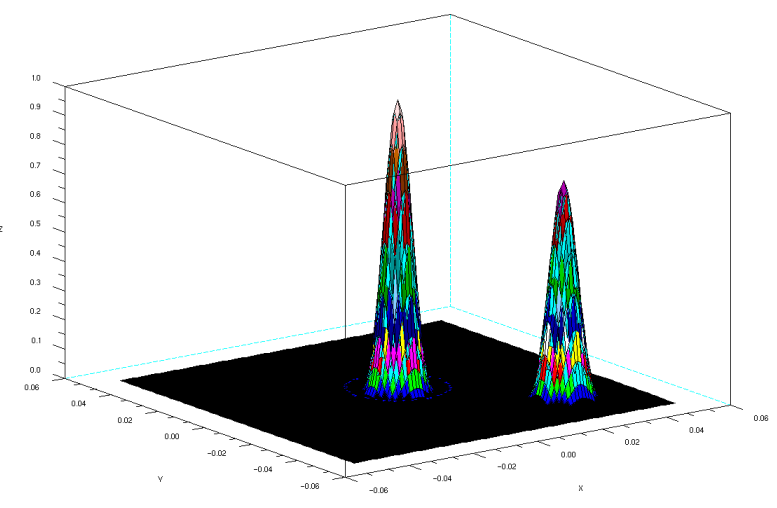

Figure 1: 3D representation of beam patterns

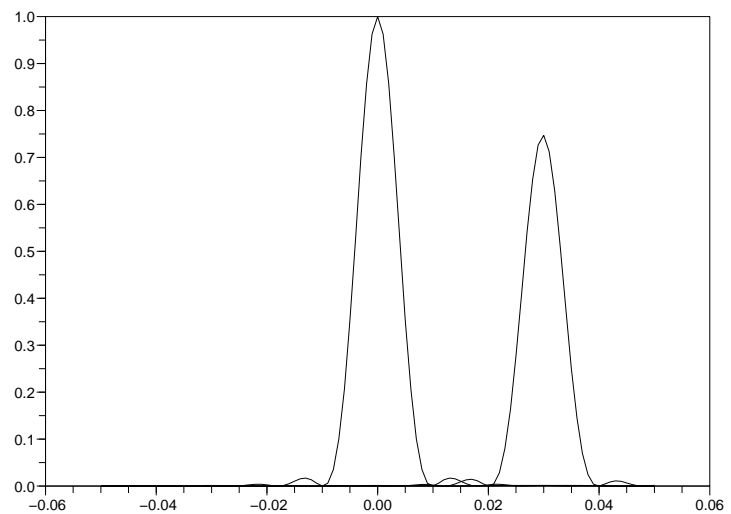

Figure 2: 2D representation of beam patterns

A minimum quality for a communication between a user and the gateway is required. It corresponds to $\left(\frac{C}{N}\right)_{R s M o d C o d}$ that depends on the modulation 
scheme and the code scheme (the digital communication scheme considered here is a phase key shifting). More precisely, a user will be served if

$$
\frac{C}{N+I} \geq\left(\frac{C}{N}\right)_{R s M o d C o d}
$$

where $\frac{C}{N+I}$ is the user Signal-to-Interference-plus-Noise Ratio (SINR) which determines quantitatively the signal quality.

The link budget enables to compute the SINR :

$$
\begin{aligned}
\left(\frac{C}{N+I}\right)^{-1}= & \left(\frac{C}{N}\right)_{F e e d e r}^{-1}+\left(\frac{C}{I}\right)_{F e e d e r}^{-1}+ \\
& \left(\frac{C}{I M}\right)^{-1}+\left(\frac{C}{N}\right)_{\text {User }}^{-1}+\left(\frac{C}{I}\right)_{\text {User }}^{-1},
\end{aligned}
$$

in which

$$
\left(\frac{C}{N}\right)_{U s e r}=K_{1} \times \frac{G_{\text {Sat }}\left(U s e r_{-} B e a m \rightarrow U s e r\right)}{K_{2}}
$$

and

$$
\left(\frac{C}{I}\right)_{U s e r}=\frac{G_{\text {Sat }}\left(U s e r_{\_} \text {Beam } \rightarrow U s e r\right)}{\sum_{j \in \text { Interf }}\left(G_{\text {Sat }}\left(U s e r_{\_} B e a m \rightarrow U \operatorname{ser}(j)\right)\right.} .
$$

Terms $K_{1}$ and $K_{2}$ involve technical parameters, such as the atmospheric loss, the antenna temperature and the Equivalent Isotropically Radiated Power (EIRP) which are considered as constant. Consequently, $\left(\frac{C}{N}\right)_{U s e r}$ and $\left(\frac{C}{I}\right)_{U s e r}$ only depends on user position and beam center position. Indeed, $G_{\text {Sat }}\left(U_{\text {ser_Beam }} \rightarrow\right.$ User $)$ describes the gain for a user terminal and its beam (see equation (1)). The set Inter $f$ is the set of users sharing the same channel. Moreover, $\left(\frac{C}{N}\right)_{\text {feeder }}$ and $\left(\frac{C}{I}\right)_{\text {feeder }}$ which are gateway characteristics, and $\left(\frac{C}{I M}\right)$ that is a satellite characteristic are also constant.

Remark 1. For simplification reasons, considerations on signal processing are not tackled. However, for the different ratios $\frac{C}{I}, \frac{C}{N}$ and $\frac{C}{N+I}$, the variables $C, I$ and $N$ mean respectively "carrier", "interferences" and "noise".

Specifications of the system are the following.

- Beams are only adaptive in direction and not in shaping (although it is technically feasible). 
- For the first scenario (involving SDMA), each user has a beam directly centered on him. It leads to consider as many beams as users in the system.

- We only focus on the case of users-to-feeder (gateway) link.

\subsection{Scenarios description}

In the users-to-feeder link, interferences can occur in the link budget when several users share the same frequency. We illustrate this phenomena in fig.3 where black diamonds are users sharing the same frequency. It shows that users $u_{3}$ and $u_{2}$ are interferers for user $u_{1}$.

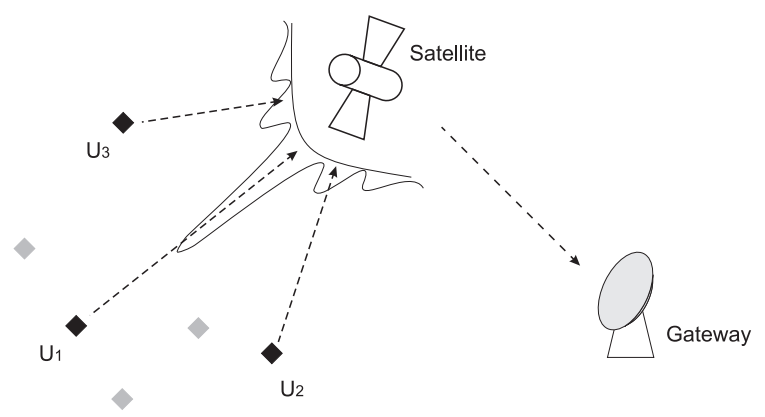

Figure 3: Users-to-feeder link.

In the first scenario, named "scenario 1", we consider that each user has a beam directly centered on him (which is possible thanks to SDMA). The number of available channels is 8 . In this case, $G_{2}\left(u, v, u_{0}, v_{0}\right)$ is always equal to the maximum value (that is $G_{2}\left(u, v, u_{0}, v_{0}\right)=1$ ) since $(u, v)=\left(u_{0}, v_{0}\right)$.

Contrary to scenario 1 , the second scenario involves fixed beams, however the frequency assignment is variable according to the demand (the channel number can be adjusted for a beam). The service area is composed of 40 fixed beams which form a spot-based coverage. We also have 8 available channels. Consequently, (since no channel can be used more than once in a spot) we can not serve more than 8 users in a spot.

For both scenario, the FAP considered in this paper consists in finding and interference-free frequency allocation to the users maximizing the number of served users. This problem is static: the set of users is known in advance and we do not take dynamic arrivals and departures of users into account. 


\section{A cumulative interference representation}

In a FAP where channels are limited, results depend on the ability of the system to allocate the same channel to several users.

In this section, we show that it is possible to obtain a cumulative representation of interferences from equations (2) and (3).

Involving the link budget, constraint (2) for a user $i$, becomes

$$
\frac{1}{A+B_{i}+\frac{\sum_{j \in \text { Interf }(i)} G_{\text {Sat }}\left(U \text { User_Beam }_{-}(i) \rightarrow U \operatorname{ser}(j)\right)}{G_{\text {Sat }}(\text { User_Beam }(i) \rightarrow U \operatorname{ser}(i))}} \geq D
$$

where

$$
\begin{gathered}
A=\left(\frac{C}{N}\right)_{\text {Feeder }}^{-1}+\left(\frac{C}{I}\right)_{\text {Feeder }}^{-1}+\left(\frac{C}{I M}\right)^{-1}, \\
B_{i}=\left(\frac{C}{N}\right)_{\text {User }_{i}}^{-1}=\frac{K_{2}}{K_{1} \times G_{\text {Sat }}\left(U \operatorname{User} \_B e a m(i) \rightarrow U \operatorname{ser}(i)\right)}
\end{gathered}
$$

and

$$
D=(C / N)_{R s M o d C o d}
$$

Previous inequation leads to

$$
\begin{aligned}
& 1 \geq A D+D B_{i}+
\end{aligned}
$$

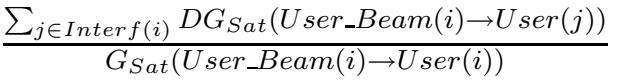

and finally, the cumulative representation follows

$$
\begin{gathered}
\sum_{j \in \operatorname{Interf}(i)} D G_{\text {Sat }}\left(U \operatorname{User}_{-} B \operatorname{Beam}(i) \rightarrow U \operatorname{ser}(j)\right) \leq \\
G_{\text {Sat }}\left(U \operatorname{User} \_B e a m(i) \rightarrow U \operatorname{ser}(i)\right)\left(1-A D-B_{i} D\right) .
\end{gathered}
$$

For scenario 1, we write last constraint

$$
\sum_{j \in \operatorname{Interf}(i)} \delta_{i j} \leq \alpha_{i}
$$

where

$\alpha_{i}=G_{\text {Sat }}\left(U \operatorname{ser} \_B e a m(i) \rightarrow U \operatorname{ser}(i)\right)\left(1-A D-B_{i} D\right)$ 
and

$\delta_{i j}=D G_{\text {Sat }}(U$ User_Beam $(i) \rightarrow U \operatorname{ser}(j))$.

Let the system with $n$ users, $\alpha$ is a $n$-row vector and $\delta$ is a $n \times n$ matrix.

We can deduce that $\alpha_{i}$ represents the maximum level of interferences that the user $i$ can support. In this way, $\delta_{i j}$ describes the interference level of user $j$ if users $j$ and $i$ share the same frequency. The cumulative representation is motivated by a linear representation (although constraint (2) was not linear) that enable the use of integer linear programming (see $\S 4.2$ ).

Concerning scenario 2, we denote the interference inequality by

$$
\sum_{j \in \operatorname{Interf}(i)} \gamma_{i j} \leq \beta_{i}
$$

It is worth emphasizing that $\alpha_{i}$ and $\beta_{i}$ are different since the center of the beam related to user $i$ does not correspond to user $i$ coordinates in scenario 2 whereas in scenario 1 , it does. The same remark can be done for $\delta_{i j}$ and $\gamma_{i j}$

\section{Modeling and solving scenarios 1 and 2 FAP}

\subsection{FAP literature overview}

Most approaches dealing with interference minimization FAP consider binary interference constraints, i.e. involving only two users. Because of the strong links between graph coloring and frequency allocation with binary interference constraints, most methods found in the literature are inspired by coloring algorithms. We also know unfortunately the graph coloring problems, and consequently the FAP, are NP-hard. Among the proposed methods, the constructive (greedy) algorithms are widely used since they are simple, fast and also able to solve dynamic FAP. In this category, we find the generalization of DSATUR procedure Brélaz (1979). Other more sophisticated algorithms, such as local search, metaheuristics, ILP and constraint programming approaches, are frequently encountered, see Resende and Pardalos (2006)Murphey et al. (1999) and Aardal et al. (2003) for a comprehensive review of the state-of-the-art methods for the FAP with binary interference constraints.

One of the difficulties appearing in the telecommunication system considered in this study (for both scenarios 1 and 2) lies in the explicit consideration of non-binary interference constraints. In terms of graph coloring, deciding 
whether a given coloring is feasible or not cannot be made anymore by checking pairwise user color assignments. Instead, for a given user, the cumulative interferences of the users assigned to the same color has to be computed. Then, the coloring is feasible if this cumulative interference remains under a user-dependent threshold (see Section 3). In the literature, only a few approaches take account explicitly of such interferences for frequency assignment Dunkin et al. (1998); Mannino and Sassano (2003); Palpant et al. (2008). This study is partly based on integer linear programming formulations proposed in Palpant et al. (2008).

\subsection{Integer linear programming formulations (ILP)}

Taking account of hypothesis and simplifications presented in Section 2, FAP corresponding to scenarios 1 and 2 is at first sight similar to coloring problems and thus formalized as the corresponding combinatorial optimization problems. Each user has to be assigned a color, representing the allocated carrier. However there are some significant differences. There cumulative interferences and the number of color is fixed while the number of assigned users must be maximized. This correspond to the MaxFAP problem in the classification described in Aardal et al. (2003).

\subsubsection{SDMA FAP (scenario 1)}

For the SDMA FAP, (scenario 1), the following data are considered. $n$ denotes the number of users. $U=\{1, \ldots, n\}$ is the set of users. $C$ is the number of colors (channels). $\alpha_{i}$ denotes the interference threshold for user $i$. $\delta_{i j}$ is the interference component from user $j$ on user $i$, if $i$ and $j$ are assigned the same color.

Binary decision variables $x_{i c}$ are defined for $i \in\{1, \ldots, n\}$ and $c \in$ $\{1, \ldots, C\}$ with $n$, the number of users and $C$ the number of available colors. $x_{i c}=1$ if color $c$ is allocated to user $i$ and $x_{i c}=0$ otherwise. The problem 
can be represented by the following ILP:

$$
\begin{aligned}
& \max \sum_{i=1}^{n} \sum_{c=1}^{C} x_{i c} \\
& \sum_{c=1}^{C} x_{i c} \leq 1 \quad i=1, \ldots, n \\
& \sum_{j=1}^{n} \delta_{i, j} x_{j c} \leq \alpha_{i}+M_{i}\left(1-x_{i c}\right) \\
& \quad i=1, \ldots, n \quad c=1, \ldots, C \\
& x_{i c} \in\{0,1\} \quad i=1, \ldots, n \quad c=1, \ldots, C
\end{aligned}
$$

Objective (6) consists in maximizing the number of accepted users. Constraints (7) state that at most one color has to be selected for each user. Constraints (8) are the cumulative interference constraints. They represent, in case color $c$ is allocated to user $i$, the respect of the threshold for user $i$ taking account of users that are assigned color $c$, i.e. possible interferers. Constant $M_{i}$ has to be large enough to withdraw the constraint if $i$ is not assigned color $c\left(x_{i c}=0\right)$. More precisely, we set $M_{i}=\sum_{j=1}^{n} \delta_{i j}-\alpha_{i}$.

\subsubsection{Fixed beam FAP (scenario 2)}

For the fixed beam FAP (scenario 2), the data are similar with additional features concerning the spots (a spot designing the area covered by a given beam): $m$ denotes the number of spots. $S=\{1, \ldots, m\}$ is the set of spots. $U_{s}$ is set of users covered by spot $s \in S$. A user $i$ belong to the set $U_{s}$ corresponding to his closest beam. $\beta_{i}$ denotes the interference threshold for user $i . \gamma_{i j}$ is the interference component from user $j$ on user $i$ if $i$ and $j$ are assigned the same color.

We define the fixed beam FAP as a combinatorial optimization problem resembling the SDMA FAP preventing two users covered by the same spot 
from being assigned the same non-zero color. We obtain the following ILP:

$$
\begin{aligned}
& \max \sum_{i=1}^{n} \sum_{c=1}^{C} x_{i c} \\
& \sum_{c=1}^{C} x_{i c} \leq 1 \quad i=1, \ldots, n \\
& \sum_{i \in U_{s}} x_{i c} \leq 1 \quad s=1, \ldots, m \quad c=1, \ldots, C \\
& \sum_{j=1}^{n} \gamma_{i, j} x_{j c} \leq \beta_{i}+N_{i}\left(1-x_{i c}\right) \\
& i=1, \ldots, n \quad c=1, \ldots, C \\
& x_{i c} \in\{0,1\} \quad i=1, \ldots, n \quad c=1, \ldots, C
\end{aligned}
$$

Scenario 2 ILP differs with the scenario 1 ILP via constraints (12) and (13). Constraints (12) prevent any color from being allocated more than once in a given spot. Hence a maximum number of $C$ users may be served in the same spot. In constraints (13), values $\beta_{i}$ and $\gamma_{i j}$ differ from $\alpha_{i}$ and $\delta_{i j}$ since beams are not centered on the users (see Section 3). As for scenario $1, N_{i}$ has to be large enough to ensure the constraint is verified when color $c$ is not allocated to user $i$. For this purpose, we set $N_{i}=\sum_{j=1}^{n} \gamma_{i j}-\beta_{i}$. Both above-defined ILP can be solved by an integer linear programming solver, via branch and bound.

\subsection{Greedy algorithms}

Solving the ILP formulations provides optimal solutions only for small problems. For large-sized problems it is necessary to use a heuristic. We propose greedy algorithms to solve scenarios 1 and 2 FAP. For both scenarios, the principle of the greedy algorithms is, first, to consider the users sequentially according to a given criterion named the user priority rule. Second, either the selected user is assigned a frequency or rejected according to a second criterion, the frequency priority rule. Let $Q$ denote the set of users that have not been assigned a color yet. Initially, we have $Q=U$. At each step of the greedy algorithm, a user $i$ is removed from $Q$ and is either rejected or assigned a color. In the following sections we described the variants proposed for each scenarios. 


\subsubsection{SDMA FAP (Scenario 1)}

For scenario 1, the principle of the greedy algorithm is summarized in Algorithm 1, where $F_{j}$ denote the frequency (color) allocated to user $j$ if $1 \leq F_{j} \leq C$ and $F_{j}=0$ indicates user $j$ is rejected.

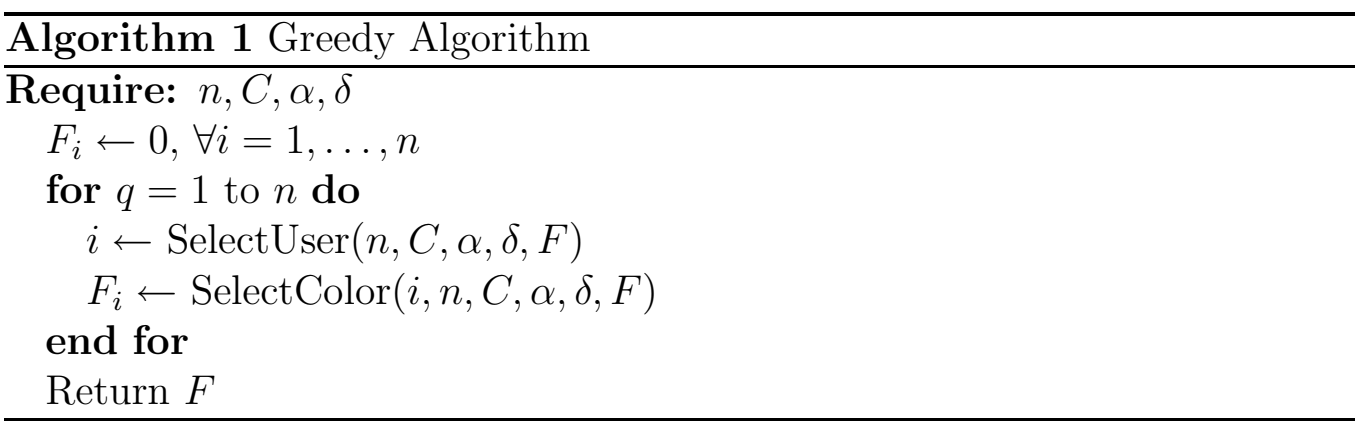

For both the user and priority rules, we may use the frequency margin, where the margin $M(i, c)$ of a user $i \in Q$ for a color $c$ is given by $M(i, c)=$ $\alpha_{i}-\sum_{j \in U \backslash Q \cup\{i\}, F_{j}=c} \delta_{i j}$. Namely, this margin corresponds to the positive or negative slack of the cumulative interference constraint for user $i$ terminal if it is assigned color $c$.

As a preliminary result, we observed that the user priority rule aiming at selecting first the most constrained users in terms of available colors, as for the well-known DSATUR algorithm for standard graph coloring problems gives bad results for this problem. In fact we were brought to consider a kind of hybrid reverse DSATUR rule, aiming a assigning users with a large number of available colors, alternating this rule with the selection of the user having maximum interferences with the previously assigned user. More precisely, we tested the two following user priority rules.

- Lexicographic. The user with the smallest number is selected.

- Hybrid. The proposed rule selects the user having the largest number of available colors, a color $c$ being available for user $i \in Q$ if $M(i, c) \geq 0$ and if for all users $j \in U \backslash Q$ having already been assigned color $c$, $M(j, c) \geq 0$. In case of a tie, we select the user having the largest total margin for all its available colors. Let $i$ denote the user selected with this rule. For the next iteration, we select the user having maximum inteference with $i$, i.e. the user $j$ maximizing $\delta_{i, j}+\delta_{j, i}$ and we alternate the two rules. 
For the frequency selection, we propose to use two priority rules:

- Lexicographic. The smallest available frequency is selected.

- Most used. The most used available frequency is selected. In case of tie we select the color $c$ that maximizes the sum of margins $M(j, c)$ for all users $j \in Q$.

The proposed greedy algorithms run in $O\left(n^{2} C\right)$ time.

\subsubsection{Fixed beam FAP (Scenario 2)}

For scenario 2, the above-described user and frequency priority rules can be applied with the notable difference that

- values $\beta_{i}$ and $\gamma_{i, j}$ are used instead of $\alpha_{i}$ and $\delta_{i, j}$, respectively.

- color availability for a user $i \in Q$ in a spot $s$ has to consider the other users in $U_{s}$. As soon as a user in $s$ has been assigned a color $c$, then $c$ becomes unavailable for the other users in $U_{s}$.

\section{Computational experiments and simulations}

The ILP formulations have been solved using IBM/ILOG CPLEX 12.1 CPLEX (2009) and the greedy algorithms have been coded in $\mathrm{C}++$. We tested the proposed algorithms with $C=8$, increasing stepwise the numbers of users by 20 from 20 to 200 users (which corresponds to reuse rates from $20 / 8=2.5$ to $200 / 8=25$ ), yielding 1000 instances. With regard to scenario 2 , we consider 40 fixed beams $(m=40)$. For each number of users, a set of 100 FAP data instances was obtained by randomly generating the user positions on the service area (uniform distribution). The results were obtained on a Intel Core 2 Duo processor with $2,93 \mathrm{GHz}$ and $1.9 \mathrm{~GB}$ RAM. The CPU time for the ILP resolution has been limited to 60 seconds afterwhich the best obtained integer solution is returned. The CPU times for the greedy algorithms were negligible.

We first present a comparison of the greedy algorithms. We selected only the algorithms performing better than the simple lexicographic rule (for user and frequency). Table 1 reports the average number of accepted users over the 1000 instances for the three best algorithms.

Fig.4 displays, for each scenario/algorithm/number of users, the average number of accepted users in the computed frequency allocation plans. For 


\begin{tabular}{lcc}
\hline & Scenario 1 & Scenario 2 \\
\hline Lexicographic (user+frequency) & 97.12 & 76.39 \\
Lexicographic (user)+ Most used (frequency) & 97.17 & 76.38 \\
hybrid (user)+Most used (frequency) & 97.31 & 77.13 \\
\hline
\end{tabular}

Table 1: Average number of accepted users over 1000 instances

the greedy algorithms we selected for each instance the best value obtained by the three best algorithms displayed in Table 1 .

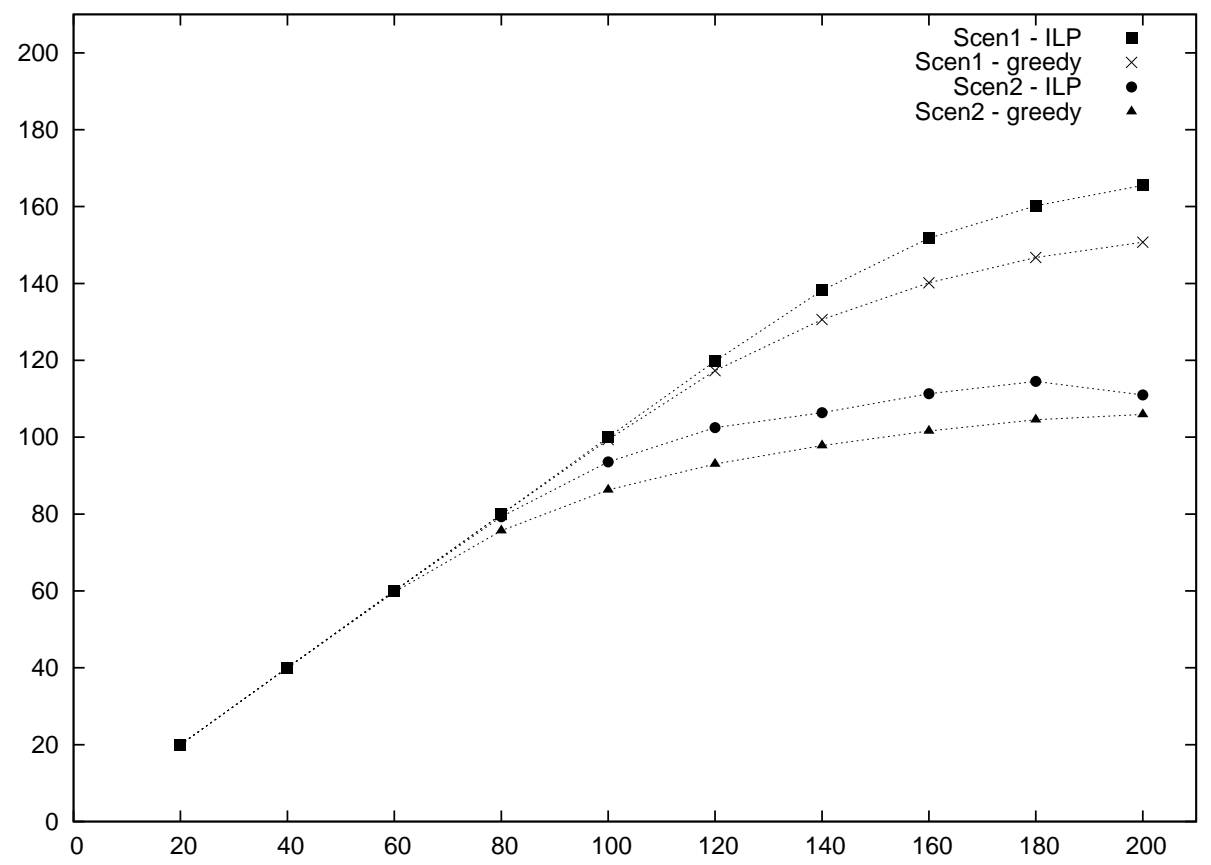

Figure 4: Average numbers of accepted users obtained by the proposed algorithms for each number of users

Last, to have a better insight on algorithm performance, we give in Fig. 5 , for each scenario/algorithm/number of users, the average gap from the upper bound obtained by ILP (as the best open node in the branch and bound tree). 


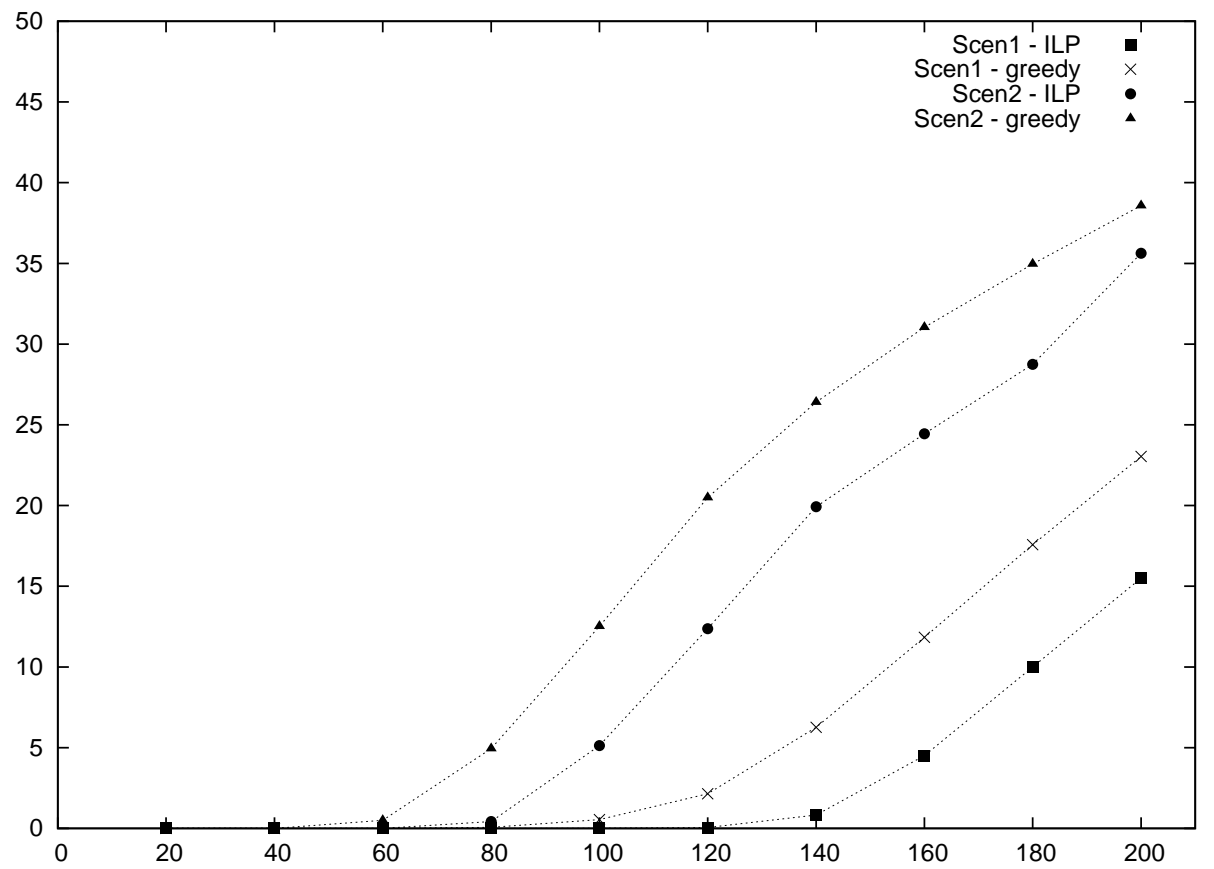

Figure 5: Average gap obtained by the proposed algorithms for each number of users

\section{Analysis}

Table 1 shows that the results of the greedy algorithm is very close. The hybrid method we propose for the user selection rule gives the best results with the most used frequency rule. However it was difficult to give better results than the simple lexicographic rules.

Fig. 4 clearly shows that the best results in terms of quality of service using the proposed algorithms are obtained for scenario 1 (SDMA-based system). ILP-based algorithms obtain significantly better results than the simple greedy algorithms for both scenarios. However, the greedy algorithm for scenario 1 performs better than the ILP-based algorithm for scenario 2.

Fig.5 shows as expected that the gap increases as the number of users increases. The gap is also more important for scenario 2 than for scenario 1. This shows that the optimization problem of scenario 1 is easier to solve than the optimization problem of scenario 2, at least for the tested methods.

This is confirmed by the ILP results in optimality terms. Indeed, Table 2 shows that the number of proved optimal solutions falls from $100 \%$ to $82 \%$ 


\begin{tabular}{lcccccccccc}
\hline$n$ & 20 & 40 & 60 & 80 & 100 & 120 & 140 & 160 & 180 & 200 \\
\hline Scen 1 - ILP & 100 & 100 & 100 & 100 & 99 & 94 & 46 & 3 & 0 & 0 \\
Scen 2 - ILP & 100 & 100 & 100 & 82 & 6 & 0 & 0 & 0 & 0 & 0 \\
\hline
\end{tabular}

Table 2: Number of optima proved by ILP

then $6 \%$ when the number of users increase from 60 to 100 for scenario 2 while this number stays above $90 \%$ of up to 120 users for scenario 3. Forbidding identical colors in the same spot makes the search of an optimal solution harder.

Furthermore, crossing Figure 4 and Table 2, we see that for instances where $100 \%$ of optima are proved for both scenarios, i.e. for instances having up to 60 users, no difference can be observed between the scenarios since all users are accepted. To rigorously compare the performance of the scenarios, we gather the instances with $n \geq 80$ and for which the optimum number of accepted users was proved by ILP for both scenarios. We obtain 63 instances of known optima in both cases. Among these 63 instances, Greedy algorithm for scenario 1 obtains solutions strictly better that ILP for scenario 2 for 24 instances. It obtains only one strictly worse solution. Hence the simple greedy algorithm for scenario 1 outperforms the optimal solutions for scenario 2 .

This numerical example with real parameters for the simulation shows qualitatively the benefits of scenario 1 even with a simple greedy algorithm. We can also note that beyond 80 users, which correspond to a reuse rate of 10, differences between algorithms increase.

\section{Conclusion}

In this paper we have developed integer linear programming formulations and greedy algorithms for frequency assignment problems involving cumulative interferences. The cumulative approach enables to take into account the non linear characteristics of interferences. Combining the SDMA system and the cumulative approach, we proved in section 6 the efficiency of scenario 1 with the two algorithms. Even better results and system optimizations using SDMA could be obtained by allowing shifts of the beam centers around the users and adjusting the EIRP parameters, yielding as a counterpart harder FAP. These features, together with consideration of dynamic aspects, constitutes the basis for further research. 


\section{References}

Aardal, K. I., van Hoesel, C. P. M., Koster, A. M. C. A., Mannino, C., Sassano, A., 2003. Models and solution techniques for the frequency assignment problem. 4OR 1 (4), 261-317.

Brélaz, D., 1979. New methods to color the vertices of a graph. Communications of the ACM 22, 251-256.

Corbel, E., Buret, I., Gayrard, J.-D., Corazza, G. E., Bolea-Alamanac, A., Aug. 2008. Hybride satellite and terrestrial mobile network for 4g: Candidate architecture and space segment dimensioning. In: ASMS. Bologna, Italy.

CPLEX, I. I., 2009. Concert Technology version 12.1 C++ API Reference Manual. IBM.

Dunkin, N. W., Bater, J. E., Jeavons, P. G., Cohen, D. A., 1998. Towards high order constraint representations for the frequency assignment problem. In: CSD-TR-98-05. Royal Holloway, University of London, Egham, Surrey, UK.

Gayrard, J.-D., Buret, I., Bolea-Alamanac, A., Sep. 2007. The role of satellites in broadband wireless access for public protection, disaster relief and gmes missions. In: 13th Ka and Broadband Communications Conference. Turin, Italy.

Liberti, J., Rappaport, T., 1999. Smart Antennas for Wireless Communications. Prentice Hall.

Mannino, C., Sassano, A., 2003. An enumerative algorithm for the frequency assignment problem. Discrete Applied Mathematics 129 (1), 155-169.

Murphey, R., Pardalos, P. M., Resende, M. G. C., 1999. Handbook of Combinatorial Optimization: Supplement Volume A. Springer, Ch. Frequency Assignment Problems.

Palpant, M., Oliva, C., Artigues, C., Michelon, P., Biha, M. D., 2008. Models and methods for frequency allocation with cumulative interference constraints. International Transactions in Operational Research 15 (3), 307324. 
Resende, M. G. C., Pardalos, P. M., 2006. Handbook of Optimization in Telecommunications. Springer. 